Analysis of demographic data revealed a significantly higher median age for patients with GII.P17_GII.17 infections (55 [SD 49.8] months) than for patients with GII.4 infections (24 [SD 13.6] months) $(\mathrm{p}<0.005 ; 2$-tailed Mann U-test, $p=0.00433$ [95\% CI 0.4-6.5]). These observations are consistent with a lack of specific herd immunity in the population, meaning that the GII.17 virus can infect older patients more easily than GII.4 viruses can, as observed in Hong Kong (4).

Our analysis indicates that, in Italy in winter 201516 , the epidemiologic pattern of norovirus GII.17 viruses markedly changed, suggesting increased circulation of the variant Kawasaki 2014 among children, although GII.4 variants (the capsid variant Sydney 2012 with the GII.Pe or GII.P4 polymerase) were still predominant. The mechanisms driving the global spread of norovirus GII.17 could include the broad range of co-receptors used by these viruses (10) or the limited cross-antigenic relationships with the predominant GII.4 strains that could trigger mechanisms of antigenic escape. Norovirus GII.17 could present a challenge for the development of norovirus vaccines because it is not clear whether, and to what extent, there is crossprotection between vaccine antigens and GII.17 viruses ( 6 ).

\section{Acknowledgments}

We thank Giuseppe Iacono and Mirella Collura for granting access to patients' files and Daniele Casulli for skilled technical assistance.

Dr. Giammanco is a clinical microbiology specialist and associate professor at the University of Palermo, Dipartimento di Scienze per la Promozione della Salute e Materno Infantile "G. D'Alessandro". His primary research interests are enteric viruses, emerging viral pathogens, viral epidemiology, and microbial typing.

\section{References}

1. Matsushima Y, Ishikawa M, Shimizu T, Komane A, Kasuo S, Shinohara M, et al. Genetic analyses of GII.17 norovirus strains in diarrheal disease outbreaks from December 2014 to March 2015 in Japan reveal a novel polymerase sequence and amino acid substitutions in the capsid region. Euro Surveill. 2015;20:21173. http://dx.doi.org/10.2807/1560-7917.ES2015.20.26.21173

2. Fu J, Ai J, Jin M, Jiang C, Zhang J, Shi C, et al. Emergence of a new GII.17 norovirus variant in patients with acute gastroenteritis in Jiangsu, China, September 2014 to March 2015. Euro Surveill. 2015;20:21157. http://dx.doi.org/10.2807/ 1560-7917.ES2015.20.24.21157

3. Lu J, Sun L, Fang L, Yang F, Mo Y, Lao J, et al. Gastroenteritis Outbreaks Caused by Norovirus GII.17, Guangdong Province, China, 2014-2015. Emerg Infect Dis. 2015;21:1240-2. http://dx.doi.org/10.3201/eid2107.150226

4. Chan MCW, Lee N, Hung T-N, Kwok K, Cheung K, Tin EKY, et al. Rapid emergence and predominance of a broadly recognizing and fast-evolving norovirus GII.17 variant in late 2014. Nat Commun. 2015;6:10061. http://dx.doi.org/10.1038/ ncomms 10061
5. Parra GI, Green KY. Genome of Emerging Norovirus GII.17, United States, 2014. Emerg Infect Dis. 2015;21:1477-9. http://dx.doi.org/10.3201/eid2108.150652

6. de Graaf M, van Beek J, Vennema H, Podkolzin AT, Hewitt J, Bucardo F, et al. Emergence of a novel GII.17 norovirus - end of the GII.4 era? Euro Surveill. 2015;20:21178. http://dx.doi.org/10.2807/1560-7917.ES2015.20.26.21178

7. Dinu S, Nagy M, Negru DG, Popovici ED, Zota L, Oprișan G. Molecular identification of emergent GII.P17-GII.17 norovirus genotype, Romania, 2015. Euro Surveill. 2016;21:30141.

8. Medici MC, Tummolo F, Calderaro A, Chironna M, Giammanco GM, De Grazia S, et al. Identification of the novel Kawasaki 2014 GII.17 human norovirus strain in Italy, 2015. Euro Surveill. 2015;20:30010. http://dx.doi.org/10.2807/1560-7917. ES.2015.20.35.30010

9. Kroneman A, Vennema H, Deforche K, v d Avoort H, Peñaranda S, Oberste MS, et al. An automated genotyping tool for enteroviruses and noroviruses. J Clin Virol. 2011;51:121-5. http://dx.doi.org/10.1016/j.jcv.2011.03.006

10. Zhang X-F, Huang Q, Long Y, Jiang X, Zhang T, Tan M, et al. An outbreak caused by GII.17 norovirus with a wide spectrum of HBGA-associated susceptibility. Sci Rep. 2015;5:17687. http://dx.doi.org/10.1038/srep17687

Address for correspondence: Giovanni Maurizio Giammanco, Department of Health Promotion Sciences and Mother and Child Care “G. D’Alessandro”, University of Palermo, Via del Vespro, 133 - 90127 Palermo, Italy; email: giovanni.giammanco@unipa.it

\title{
Disseminated Mycobacterium genavense Infection in Patient with Adult-Onset Immunodeficiency
}

\section{Takanori Asakura, Ho Namkoong, Takuro Sakagami, Naoki Hasegawa, Kiyofumi Ohkusu, Akira Nakamura}

Author affiliations: Japan Society for the Promotion of Science, Tokyo, Japan (T. Asakura); Keio University School of Medicine, Tokyo (T. Asakura, H. Namkoong, N. Hasegawa); Eiju General Hospital, Tokyo (H. Namkoong); Niigata University Graduate School of Medical and Dental Sciences, Niigata, Japan (T. Sakagami); Tokyo Medical University, Tokyo (K. Ohkusu); Asahi General Hospital, Chiba, Japan (A. Nakamura)

DOI: https://dx.doi.org/10.3201/eid2307.161677

We report a case of disseminated Mycobacterium genavense infection resulting from neutralizing anti-interferon-y autoantibodies in the patient. We identified $M$. genavense targeting the hsp65 gene in an aspiration specimen of the 
lymph node. Adult-onset immunodeficiency caused by neutralizing anti-interferon- $y$ autoantibodies, in addition to HIV infection, can lead to disseminated nontuberculous mycobacterial infection.

$M$ ycobacterium genavense is a ubiquitous nontuberculous mycobacteria (NTM), first described as a human infection in the 1990 s as a primary cause of fatal disseminated infection in HIV-infected patients with low CD4 counts (1). M. genavense also is recognized as an opportunistic pathogen in patients without HIV who have severe immunodeficiency, such as that after solid-organ or hematopoietic stem cell transplantation or immunosuppressive therapy $(2,3)$. The diagnosis of $M$. genavense infection is clinically challenging because of the difficulties in routinely culturing the organism and the absence of specific symptoms, even in fatal infections. Therefore, diagnosing $M$. genavense infection in patients without known evidence of immunodeficiency is particularly difficult. We report a previously healthy 66-year-old man with multiple lymphadenopathies in whom disseminated $M$. genavense infection resulting from the presence of neutralizing antiinterferon- $\gamma$ (anti-IFN- $\gamma$ ) autoantibodies was diagnosed.

In November 2015, the patient sought care at Asahi General Hospital (Chiba, Japan) for a 2-week history of right-side neck swelling and abdominal pain. His vital signs were within reference ranges. Except for right cervical lymphadenopathy, findings on physical examination were unremarkable. HIV antibodies were undetectable, and CD4/CD8 lymphocyte counts were within reference ranges. No mediastinal or lung involvement was detected on chest computed tomography (CT) scan. Gallium-67 singlephoton emission CT/CT revealed high-intensity accumulation of the right cervical and ileocolic lymph nodes (online Technical Appendix Figure, https://wwwnc.cdc.gov/ EID/article/23/7/16-1677-Techapp1.pdf). Acid-fast bacilli (AFB) staining of the lymph-node aspiration specimen yielded positive results; however, findings on solid media culture and PCR for detecting M. tuberculosis, M. avium, and M. intracellulare were negative. After a 6-week outpatient follow-up, the patient returned with newly developed right axillary lymphadenopathy. An aspiration specimen of the lymph node showed positive AFB staining and was submitted for molecular biologic analysis. M. genavense was identified on amplification and sequencing analysis targeting the hsp65 gene (4). We strongly suspected neutralizing anti-IFN- $\gamma$ autoantibodies as the cause because the whole blood IFN- $\gamma$ level with mitogen stimulation was low, as determined using an IFN- $\gamma$-releasing assay (QuantiFERON TB 3G; Cellestis, Carnagie, VIC, Australia). A high serum-neutralizing anti-IFN- $\gamma$ autoantibody titer and inhibited STAT1 (signal transducer and activator of transcription 1) phosphorylation through IFN- $\gamma$ stimulation in the leukocytes were confirmed, leading to a diagnosis of disseminated $M$. genavense infection. Clarithromycin, ethambutol, rifampin, and amikacin were administered. Lymphadenopathy improved after 6 weeks, and amikacin was discontinued. No relapse occurred during 16 months of treatment.

Recent studies have described disseminated NTM infection in patients in Asia with adult-onset immunodeficiency resulting from neutralizing anti-IFN- $\gamma$ autoantibodies (5-7). Disseminated infection mainly involves the lymph nodes, followed by the osteoarticular system, bone, lungs, and skin $(6,7)$. The pathogen comprises rapidly and slowly growing mycobacteria; $M$. avium complex and $M$. abscessus are the most frequently detected. Although the long-term outcome is unclear, most patients need long-term antimicrobial therapy, and some relapses occur after treatment discontinuation $(6,7)$. Adjuvant rituximab therapy has been used for refractory disease (8).

Although disseminated $M$. genavense infections formerly only were known to occur in HIV-infected patients, the epidemiologic shift to infections in patients without HIV reflects the introduction of combination antiretroviral therapy and increasing use of immunosuppressive agents (2). In 2 previous series comprising 14 HIV-negative patients with $M$. genavense infection, most patients had known evidence of immunodeficiency; of the 12 patients treated with immunosuppressive agents, 5 had sarcoidosis, 5 were solid-organ transplantation recipients, 1 had nonHodgkin lymphoma, and 1 had rheumatoid arthritis. Only 2 patients were identified with adult-onset innate immunodeficiency $(2,3)$; 1 patient had innate interleukin-12 receptor deficiency and 1 had idiopathic CD4 lymphocytopenia.

Needle aspirates and tissue biopsy provide higher NTM diagnostic yields than does swab sampling but are insufficiently sensitive. Therefore, less frequently encountered mycobacterial species are identified by gene sequencing, reverse hybridization, and high-performance liquid chromatography (9). Moreover, the identification of $M$. genavense infection using standard mycobacterial culture methods is difficult. Acidified solid media testing with blood and charcoal is probably the most suitable method (10); however, accurate diagnosis requires additional molecular biologic analysis, such as amplification and sequencing of the $16 \mathrm{~S}$ ribosomal RNA, $h s p 65$, or $r p o B$ genes. In this case, we identified $M$. genavense using a direct molecular biologic method for aspiration specimens from the lymph node.

Little is known about death among HIV-negative patients with $M$. genavense infection, although some patients reportedly have died $(2,3)$. Although their conditions eventually improve, despite a lack of early identification of $M$. genavense, delayed diagnosis might influence death. Direct molecular biologic methods could better identify $M$. genavense infection and improve prognosis. 
We report a case of disseminated $M$. genavense infection resulting from neutralizing anti-IFN- $\gamma$ autoantibodies in the patient. M. genavense infection should be considered in the differential diagnosis of mycobacteria detected with AFB staining but not with culture, even in patients without known evidence of immunodeficiency. Adult-onset immunodeficiency acquired by neutralizing anti-IFN- $\gamma$ autoantibodies, in addition to HIV infection, can lead to disseminated NTM infection.

Dr. Asakura is a medical doctor in the Division of Pulmonary Medicine, Department of Medicine, Keio University School of Medicine, in Tokyo, Japan. His research interests include nontuberculous mycobacterial infection and non-cystic fibrosis bronchiectasis.

\section{References}

1. Bessesen MT, Shlay J, Stone-Venohr B, Cohn DL, Reves RR. Disseminated Mycobacterium genavense infection: clinical and microbiological features and response to therapy. AIDS. 1993; 7:1357-61. http://dx.doi.org/10.1097/00002030-199310000-00009

2. Hoefsloot W, van Ingen J, Peters EJ, Magis-Escurra C, Dekhuijzen PN, Boeree MJ, et al. Mycobacterium genavense in the Netherlands: an opportunistic pathogen in HIV and non-HIV immunocompromised patients. An observational study in 14 cases. Clin Microbiol Infect. 2013;19:432-7. http://dx.doi.org/10.1111/ j.1469-0691.2012.03817.x

3. Charles P, Lortholary O, Dechartres A, Doustdar F, Viard JP, Lecuit M, et al.; French Mycobacterium genavense Study Group. Mycobacterium genavense infections: a retrospective multicenter study in France, 1996-2007. Medicine (Baltimore). 2011;90:22330. http://dx.doi.org/10.1097/MD.0b013e318225ab89

4. Pai S, Esen N, Pan X, Musser JM. Routine rapid Mycobacterium species assignment based on species-specific allelic variation in the 65-kilodalton heat shock protein gene (hsp65). Arch Pathol Lab Med. 1997;121:859-64.

5. Browne SK, Burbelo PD, Chetchotisakd P, Suputtamongkol Y, Kiertiburanakul S, Shaw PA, et al. Adult-onset immunodeficiency in Thailand and Taiwan. N Engl J Med. 2012;367:725-34. http://dx.doi.org/10.1056/NEJMoa1111160

6. Chi CY, Lin CH, Ho MW, Ding JY, Huang WC, Shih HP, et al. Clinical manifestations, course, and outcome of patients with neutralizing anti-interferon- $\gamma$ autoantibodies and disseminated nontuberculous mycobacterial infections. Medicine (Baltimore). 2016;95:e3927. http://dx.doi.org/10.1097/ MD.0000000000003927

7. Valour F, Perpoint T, Sénéchal A, Kong XF, Bustamante J, Ferry T, et al.; Lyon TB Study Group. Interferon- $\gamma$ autoantibodies as predisposing factor for nontuberculous mycobacterial infection. Emerg Infect Dis. 2016;22:1124-6. http://dx.doi.org/10.3201/ eid2206.151860

8. Browne SK, Zaman R, Sampaio EP, Jutivorakool K, Rosen LB, Ding L, et al. Anti-CD20 (rituximab) therapy for anti-IFN- $\gamma$ autoantibody-associated nontuberculous mycobacterial infection. Blood. 2012;119:3933-9. http://dx.doi.org/10.1182/ blood-2011-12-395707

9. Piersimoni C, Scarparo C. Extrapulmonary infections associated with nontuberculous mycobacteria in immunocompetent persons. Emerg Infect Dis. 2009;15:1351-8, quiz 1544. http://dx.doi.org/ 10.3201/eid1509.081259

10. Realini L, De Ridder K, Hirschel B, Portaels F. Blood and charcoal added to acidified agar media promote the growth of
Mycobacterium genavense. Diagn Microbiol Infect Dis.

1999;34:45-50. http://dx.doi.org/10.1016/S0732-8893(99)00014-0

Address for correspondence: Akira Nakamura, Department of Internal Medicine, Asahi General Hospital, 1326 I, Asahi, Chiba 289-2511, Japan; email: zap11674@nifty.ne.jp; or Takanori Asakura, Division of Pulmonary Medicine, Department of Medicine, Keio University School of Medicine,, Shinanomachi 35, Shinjuku, Tokyo 160-8582, Japan; email: takanori.asakura@gmail.com

\section{Live Cell Therapy as Potential Risk Factor for $\mathbf{Q}$ Fever}

\author{
Maja George, Andreas Reich, Klaus Cussler, \\ Herrmann Jehl, Florian Burckhardt
}

Author affiliations: Robert Koch Institute, Berlin, Germany

(M. George); European Centre for Disease Prevention and Control, Stockholm, Sweden (M. George); Institute for Infectious Disease Prevention Landau, Landau, Germany (M. George, F. Burckhardt); Bavarian Health and Food Safety Authority, Oberschleissheim, Germany (A. Reich); Paul Ehrlich Institut, Langen, Germany (K. Cussler); Health Department of the Bad Duerckheim District, Bad Duerkheim, Germany $(\mathrm{H}$. Jehl)

DOI: http://dx.doi.org/10.3201/eid2307.161693

During an outbreak of $Q$ fever in Germany, we identified an infected sheep flock from which animals were routinely used as a source for life cell therapy (LCT), the injection of fetal cells or cell extracts from sheep into humans. $Q$ fever developed in 7 LCT recipients from Canada, Germany, and the United States.

Gram-negative intracellular bacteria (Coxiella burnetii) in livestock and humans. Typically, human patients show signs and symptoms, such as fever, severe headache, nausea, pneumonia, or hepatitis, 2-3 weeks after infection. Chronic Q fever develops in $\approx 1 \%-5 \%$ of patients ( 1 ).

On August 5, 2014, a local health department in the Federal State of the Rhineland Palatinate in southern Germany alerted the Federal State Agency for Consumer and Health Protection (FSACHP) (Landau, Germany) after detecting a cluster of 8 patients with pneumonia in a rural community during a 6 -week period. The local health department and FSACHP started a joint outbreak 